\title{
Comparison of Traditional Approach for Edge Detection with Soft Computing Approach
}

\author{
Neha S Joshi \\ M.Tech Student (CE) \\ Computer Department, MPSTME \\ NMIMS, Shirpur, Dist :Dhule, Maharashtra, \\ India
}

\author{
Nitin S Choubey \\ M.Tech Student (CE) \\ Computer Department, MPSTME \\ NMIMS, Shirpur, Dist :Dhule, Maharashtra, \\ India
}

\begin{abstract}
Image processing supports applications in different fields such as medicine, astronomy, product quality, industrial applications. Edge detection plays important role in segmentation and object identification process. Soft computing approach represents a good mathematical framework to deal with uncertainty of information. The performance of the well-known edge detectors, like Canny, Sobel, etc, depends critically on the choice of the input parameters. Threshold decision is the key uncertainty in the edge detection algorithms. In this paper, an improved edge detection algorithm based on fuzzy combination of mathematical morphology and multiscale wavelet transform is proposed. The proposed method overcomes the limitation of wavelet based edge detection and mathematical morphology based edge detection in noisy images. Method present will give best results for noisy images.
\end{abstract}

\section{General Terms}

Soft Computing, Image processing, Fuzzy Logic.

\section{Keywords}

Edge detection, Wavelet transform, Mathematical morphology, Fuzzy logic

\section{INTRODUCTION}

Soft computing was introduced by Lotfi A. Zadeh of the University of California, Berkley, U.S.A. in 1965[14]. Soft Computing paradigm includes fuzzy logic, neural computing, machine learning and probabilistic reasoning. Soft computing is applied for solving real world problems. It is tolerant of imprecision, uncertainty, partial truth, and approximation. Soft computing tries to develop intelligence and aims to bulid a machine which can work like human. Human like system are required as the human systems are adaptable to change and under unknown circumstances. Soft computing differs from conventional computing[15]. Although Soft computing has various advantages it suffers from the problem of large computations. System used for solving real world problem requires intelligence and be able to take decision and give reasons to it. Soft computing has several constituents[15]. The principal constituents of Soft Computing (SC) are

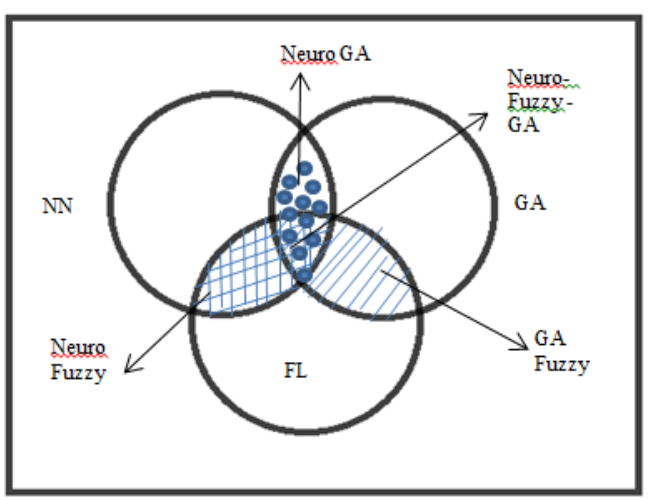

Fig 1. Soft Computing paradigm[31]

Image processing is a form of signal processing in which image is given as an input that may in video form or photograph form or it may be a video frame. Output from any image processing. can be either an image or a set of parameters of that image

Image processing mainly deals with the change in the nature of the digital image using computer in order to improve its pictorial information or make it more suitable for autonomous machine perception. Pictorial information is improved for real life applications in which human interpretation is required. Image processing supports applications in different fields such as medicine, astronomy, product quality, industrial applications. In the area of Image processing, there is uncertainty in data or task or result[11]. Reasons to uncertainty can be the randomness or vagueness or inherent ambiguity in image data. Uncertainty arises due to lot ambiguity in the area of image processing. The reasons for ambiguity can be due to image resolution, image segmentation, object recognition, scene analysis. It is required to design an algorithm which deals with such types of uncertainty. Soft computing allows to model uncertainty. Soft computing is widely being used in image processing for applications involving image compression, image segmentation, image enhancement, image transformation, image extraction, image classification, image retrieval morphology, noise reduction and edge detection. The principle of soft computing leads to near to optimum results. Soft computing can deal with the ambiguity and uncertainty in image processing in better way as compared to the traditional approaches. When soft computing is used for edge detection gives better result compare to the classical approach. The classical techniques like Sobel, Prewitt, Roberts, Canny edge detector have limitations of using fixed value of parameters or threshold. While the nature of edges is not constant due to 
which few edges left by being detected. Fuzzy logic a branch of soft computing provides us flexibility by allowing the values without any such restrictions. Fuzzy logic have IFTHEN rules and have simple structure to implement. Simply by changing or adding few more fuzzy rules result can be changed and some techniques are knowledge based in which training is required. Fuzzy logic is a logical system which is an extension of multi valued logic. It is a propositional calculus in which there are more than two truth values [31]. By using fuzzy techniques edges having different thickness can also be detected [31].Fuzzy logic is conceptually easy to understand and is flexible and is tolerant of imprecise data. Fuzzy logic is to map an input space to an output space and for doing this a list of if then statements called rules are evaluated in parallel. These Rules are useful because they use variables and adjectives that describes those variables[13].In this paper we present an algorithm for edge detection using multiscale wavelet transform and morphological operation using fuzzy rules. In this way we had utilized the advantages of wavelet transform, mathematical morphology and fuzzy systems. Results show that the proposed algorithm works well in noise condition and detect more edges.

In this paper Multiscale Wavelet Transform is used for performing smoothing on image and DWT (Discrete Wavelet Transform) is discussed for comparison with proposed method. The logic behind that discrete wavelet transform (DWT) is that it decomposes the images into sub-images. The 2-D DWT is an extension of the 1-D DWT in both the horizontal and the vertical direction.

This paper has sections as follows, section II gives Literature Survey of Edge detection Techniques and section III describes comparative method section IV gives the proposed work for system. Experimental results and discussion is discussed in Section V followed by the conclusion.

\section{RELATED WORK}

In the field of image processing Initially Canny [5] detection under noisy conditions and was also capable of detecting weak and strong edges. Further many researchers have worked in the same area. Zhao Yu-qian et al. [25] proposed a algorithm for detection. Which proved more efficient than the traditional operators LoG and Sobel. J Patel and et al. in [26] has given an algorithm in which these traditional approaches of Sobel and Lapalacianof Gaussian(LoG) are used with fuzzy system and fuzzy rules. In this approach first edges are calculated and then that result is applied to fuzzy system. This method detects multiple responses to single edge and reduces false detection. Abdallah A. Alshennawy and Ayman A. Aly [27] has given a fuzzy logic technique for edge detection without determining the threshold value. This technique gives straight lines for the lines which are straight also smoothness was the result and when this algorithm was compared with Sobel it had given better result because number of double edges detected are less as that with Sobel. In both techniques[26],[27] ground truth evaluation is not considered. Later Pushpajit A. Khaire and Nilesh singh V. Thakur [28],[29] introduced an soft computing approach based on fuzzy logic in which the results are compared with canny using ground truth of respective image[28]. Liang and Looney introduced a competitive fuzzy edge detection (CFED) method [30]. These methods divides types of edges in six patterns and fuzzy classifier is being used for finding that under which pattern edge lies. CFED which generates speckle noise. Arpit Singhal and Mandeep Singh[23] has shown a mathematical morphology noise removal cum edge detection algorithm to remove speckle noise and find edges. Parameters used are Signal to noise ratio (SNR), correlation, Structural similarity index(MSSIM), Root mean square error (RMSE) and Edge preserving index (EPI).fuzzy approach is not used here. Fabrizio Russo[13]has introduced a new approach which adopts fuzzy reasoning for edge detection without being deceived by the noise. Noise protected operator developed in this algorithm combines effective rules for edge detection and noise cancellation in same structure. .Mrs.Abhradita Deepak Borkar and Mr.Mithilesh Atulkar[16] developed fuzzy inference system which have two fuzzy sets during input and three fuzzy set during output which can detect edges for fuzzy set and further middle of maximum method is used for defuzzification on the output image. SamanSinaie, Afshin Ghanizadeh,and Siti Mariyam Shamsuddin and Emadaldin Mozafari [32] has introduced method uses fuzzy set theory and then output image is enhanced using Cellular learning Automata(CLA). because of the neighbor-considering nature has been used in CLA. In this approach $w \times w$ window size is used to divide the original image. Using fuzzy set membership function is found. Enhacement of edges is done with the help of CLA and a set of rules.Dhiraj kumar Patel and S A More[33][34] has introduced a hybrid method which uses fuzzy logic and CLA. In both papers a 10 step algorithm is proposed. In which edge is detected and edge enhancement is done. CLA is used for improving the quality of the image[34]. CLA is used for enhancement of the edges and to detect the grey level changes of neighbors of every pixel, and to detect the edge by using the changing regular of one-order or two-order directional differential coefficient. but to detect the edges CLA is used to realize improve image detection and fuzzy enhancement is mentioned. [33]. Edge detection using fuzzy logic have large computation and also faces the problem of noise[35]. Soft computing approaches are applied for edge detection earlier but there are limitations to it as like continuous edge are not obtained, etc. More improvement can be done to improve the performance of the system[36].

\section{DISCRETE WAVELET TRANSFORM}

Significant intensity changes in an image normally occur at different spatial resolution or scales. Conventional edge detectors select a special spatial mask that detects edges at a particular resolution. A small mask edge detector is susceptible to noise and produces spurious edges. to that a large mask edge detector is relatively robust to noise, but distorts the edges and may not detect some finer details[39]. Thus it is very difficult to detect edges with a single spatial edge mask[1]. The edge preserved denoising lends itself to a wavelet-based procedure for edge detection. The edge in a signal gives rise to peaks in the high pass filter outputs or the detail sub bands at concordant locations. This is a characteristic of the DWT. In other words, edges give rise to peaks across several levels of details at coordinate values that moves to the left by a factor of one half at every transition from a finer scale to a coarser scale. The stronger the edge, the higher are the peaks in the DWT. Consequently, an edge can be found from the wavelet transform by identifying peaks at concordant locations. Single level 2D discrete wavelet transform is used for the purpose of experimentation. In implementation part dwt 2 command is used. dwt 2 command performs a single-level two-dimensional wavelet decomposition with respect to either a particular wavelet which is specified. $\mathrm{db} 2$ wavelet was used for specification.

\section{PROPOSED WORK}

Wavelet and mathematical morphology[1] is used in proposed method. Wavelet transform[21] and mathematical 
morphology with fuzzy rules[6] is used in proposed method. Multi-scale wavelet transform is used for smoothing the image. Image after being smoothen is given as a input to the mathematical morphology[1] which is used as an input. Further these three output from wavelet as well as mathematical morphology are given as an input to the fuzzy inference system[16]. Where the fuzzy inference system is built by with the scheme of three inputs and one output. We have used multi scale wavelet transform[22] instead of Mallet wavelet transform[38]. Mathematical morphology[1] is applied on the smoothen image obtained by applying wavelet transform. Fuzzy inference system(FIS) is designed. There are two types of fuzzy inference system which differs by the consequent rules. The role of FIS is to take decision on the basis of the fuzzy IF-THEN" rules generated. We have used Mamdani inference system[16]. In the proposed method fuzzy rules are applied on the three images pixels that are fuzzy system input images. We have used trapezoidal membership function. Membership functions of the input images are as shown in fig.2. Equations (1)[37] and (2)[37].

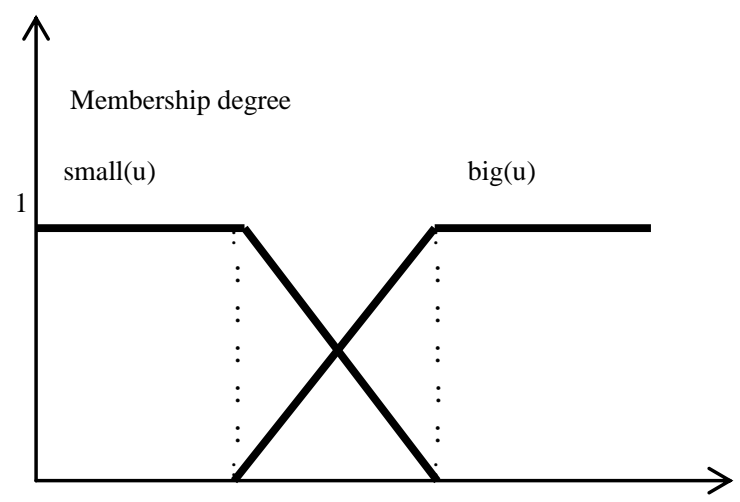

Fig. 2 The trapezoid fuzzy membership functions[37]

$\operatorname{small}(u)=\left\{\begin{array}{cr}1, & u<m \\ \frac{u-n}{m-n}, & m \leq u<n \\ 0, & u \geq n\end{array}\right.$

$\operatorname{big}(u)=\left\{\begin{array}{cr}0, & u<m \\ \frac{u-m}{n-m}, & m \leq u<n \\ 0, & u \geq n\end{array}\right.$

\subsection{Mathematical morphology}

Mathematical morphology theory [9] has been utilized as the basic theory of many newly proposed edge detectors for distinguishing contour features in an image. In this work, we utilize a morphological top-hat contrast enhancement operator to increase the pixel intensity differences in the image. Therefore, we can obtain an edge image with better quality using a morphological dilation residue edge detector. The edge image is divided into several sub-images using the quaddecomposition technique proposed by Samet et al. [10]. The pixel intensity differences in each sub-image are enhanced by means of referring to the mean and standard deviation of the edge strength in the corresponding sub-image. Since the entire fine contour features are enhanced, a global threshold is then applied to transform the edge image into a binary image. To increase the contrast level of the original image $\mathrm{F}$, the morphological contrast enhancement process is applied to $\mathrm{F}$ to increase the pixel intensity differences cooperating with a star structuring element. The contrast enhancement is

$$
F_{c}=F+W T H(F)-B T H(F)
$$

Here, $\mathrm{F}$ is the original image. Where $\mathrm{Fc}$ is the contrastenhanced image derived from the original image $\mathrm{F}$, and $\mathrm{WTH}(\mathrm{F})$ and $\mathrm{BTH}(\mathrm{F})$ are the white and the black top-hat transformations of original image $\mathrm{F}$, respectively. The definitions of operations $\mathrm{WTH}(\mathrm{F})$ and $\mathrm{BTH}(\mathrm{F})$ are given as

$$
\begin{aligned}
& \operatorname{WTH}(F)=F-(F o B) \\
& \operatorname{BTH}(\mathrm{F})=(\mathrm{F} \cdot \mathrm{B})-\mathrm{F}
\end{aligned}
$$

where the operators $\mathrm{o}$ and $\cdot$ are the mathematical morphological opening and closing operators. The primary goal of the contrast enhancement process is to accentuate and sharpen the features in the image. This process makes the original image more useful for visualization, and also makes it easier to perform post processing of the image[1],[9].

\subsection{Multi scale wavelet transform}

Wavelet is localized in time and frequency domains, so modulus maxima correspond to singularities which can be found through detecting modulus maxima. Image multi-scale edge detection can be carried out by detecting signal singularities of wavelet transform. Given two-dimensional differentiable smooth function is $\theta(\mathrm{x}, \mathrm{y})$, two requirements should be met[22].

$$
\begin{gathered}
\iint_{R} \theta(x, y) d x d y=1 \\
\lim _{x, y \rightarrow \infty} \theta(x, y)=0
\end{gathered}
$$

First-order partial derivatives of smooth function $\theta(x, y)$ in the directions of $\mathrm{x}$ and $\mathrm{y}$ should be the basic wavelet function [22].

$$
\left\{\begin{array}{l}
\Psi^{1}(x, y)=\frac{\partial \theta(x, y)}{\partial x} \\
\Psi^{2}(x, y)=\frac{\partial \theta(x, y)}{\partial y}
\end{array}\right.
$$

It can be resulted according to above equation,

$$
\left\{\begin{array}{l}
\iint_{R} \Psi^{1}(x, y) d x d y=\int_{R}[\theta(+\infty, y)-\theta(-\infty, y)] d y=0 \\
\iint_{R} \Psi^{2}(x, y) d x d y=\int_{R}[\theta(x,+\infty)-\theta(x,-\infty)] d x=0
\end{array}\right.
$$

So, $\Psi^{1}(x, y), \quad \Psi^{2}(x, y)$ is two dimensional wavelet.

Wavelet basic function can be created by basis wavelet shrinkage [22]. 


$$
\left\{\begin{array}{l}
\Psi_{a}^{1}(x, y)=\frac{1}{\alpha^{2}} \Psi^{1}\left(\frac{x}{\alpha}, \frac{y}{\alpha}\right)=\frac{\partial \theta_{\alpha}(x, y)}{\partial x} \\
\Psi_{\alpha}^{2}(x, y)=\frac{1}{\alpha^{2}} \Psi^{2}\left(\frac{x}{\alpha}, \frac{y}{\alpha}\right)=\frac{\partial \theta_{\alpha}(x, y)}{\partial y}
\end{array}\right.
$$

Where, $\theta_{\alpha}(\mathrm{x}, \mathrm{y})=\frac{1}{\alpha^{2}} \theta\left(\frac{\mathrm{x}}{\alpha}, \frac{\mathrm{y}}{\alpha}\right)$ 。

In discrete algorithm, only scale $\alpha=2 \mathrm{j}, \mathrm{j} € \mathrm{Z}$, should be considered, here, wavelet is translated into dyadic wavelet. Image $f(x, y)$ dyadic wavelet is transformed: [22]

$$
\begin{aligned}
{\left[\begin{array}{l}
\mathrm{W}_{2^{\mathrm{j}}}^{1} \mathrm{f}(\mathrm{x}, \mathrm{y}) \\
\mathrm{W}_{2^{\mathrm{j}}}^{2} \mathrm{f}(\mathrm{x}, \mathrm{y})
\end{array}\right]=} & {\left[\begin{array}{l}
\mathrm{f}(\mathrm{x}, \mathrm{y}) * \Psi_{2^{\mathrm{j}}}^{1}(\mathrm{x}, \mathrm{y}) \\
\mathrm{f}(\mathrm{x}, \mathrm{y}) * \Psi_{2^{\mathrm{j}}}^{2}(\mathrm{x}, \mathrm{y})
\end{array}\right] } \\
& =2^{\mathrm{j}}\left[\begin{array}{c}
\frac{\partial}{\partial \mathrm{x}}\left[\mathrm{f}(\mathrm{x}, \mathrm{y}) * \theta_{2^{\mathrm{j}}}(\mathrm{x}, \mathrm{y})\right] \\
\frac{\partial}{\partial \mathrm{y}}\left[\mathrm{f}(\mathrm{x}, \mathrm{y}) * \theta_{2^{\mathrm{j}}}(\mathrm{x}, \mathrm{y})\right]
\end{array}\right] \\
& =\operatorname{grad}\left|f(x, y) * \theta_{2^{j}}(x, y)\right|
\end{aligned}
$$

In the formula, $\mathrm{W}_{2 \mathrm{j}}^{1} \mathrm{f}(\mathrm{x}, \mathrm{y})$ represents horizontal detail components with scale $2 \mathrm{j}, \mathrm{W}^{2}{ }_{2 \mathrm{j}} \mathrm{f}(\mathrm{x}, \mathrm{y})$ represents vertical detail components with scale $2 \mathrm{j}$. This formula shows that wavelet transform of subjective concepts. There are different possibilities for edge detection based on fuzzy logic. In case of edge detection uncertainty is high in neighborhood pixel. One method is to define a membership function and using IFTHEN rules general edge detections can be performed. Membership function is determined heuristically. It is fast but has some limitations. Fuzzy clustering, fuzzy rule based image in the directions of row and line is the grads after smoothing when images passes smooth function, so edge points are the modulus maxima of result function. Modulus of gradient vector can be derived through formula[22].

$M_{j} f(x, y)=\sqrt{\left[W_{2}^{1} j f(x, y)\right]^{2}+\left[W_{2}^{2} j f(x, y)\right]^{2}}$

The angle between gradient vector and horizontal direction [22].

$$
A_{j} f(x, y)=\arctan \left[\frac{W_{2^{j}}^{2} f(x, y)}{W_{2^{j}}^{1} f(x, y)}\right]
$$

Local modulus maxima creation: in the direction of gradient vector, process the detection in some area, keep the maxima and delete non-maxima. Therefore, for the image pixel $(x, y)$, modulus $\mathrm{Mj} f(x, y)$ in the angle direction $\mathrm{Aj} f(x, y)$ is compared with the one of two adjacent pixels so as to judge if $\mathrm{Aj} \mathrm{f}(\mathrm{x}, \mathrm{y})$ is the local maximum and determine candidate edge point and then modulus maximum image.

\subsection{Fuzzy Logic}

Image processing possesses vagueness and ambiguity and fuzzy deals with the data uncertainty [11]. Fuzzy logic provides mathematical framework. Fuzzy methodology is generated to deal with brightness, edges and geometric features. Fuzzy logic also deals with subjective concepts. There are different possibilities for edge detection based on fuzzy logic. In case of edge detection uncertainty is high in neighbourhood pixel. One method is to define a membership function and using IF-THEN rules general edge detections can be performed. Membership function is determined heuristically. It is fast but has some limitations. Fuzzy clustering, fuzzy rule based system, fuzzy set theory are the methods of edge detection based on fuzzy logic[11]. A fuzzy inference system consists of a set of if-then rules defined over fuzzy sets. Fuzzy sets generalize the concept of a traditional set by allowing the membership degree to be any value between 0 and 1 .

\section{RESULTS AND DISCUSIONS}

Experimentation was carried on MATLAB R2010a for images. Performance parameters used are PSNR and Entropy. Proposed algorithm, the results are shown and compared to famous edge detection methods such as Sobel, Prewitt, Robert, Canny $[5,18]$ and with DWT. PSNR and Entropy were used for objective analysis. Table 2 presents comparison of algorithms using PSNR (Peak Signal to Noise Ratio) and Entropy. Simulation results show that the proposed algorithm works better than the existing algorithm. High value of PSNR shows that the edge detected image consist less noise as compared to the traditional operators. Entropy is the statistical measure Table 1 shows graphical comparison of the simulation result. By subjective method comparing result on image is shown in Fig.4. Comparison of the proposed method, traditional methods and DWT is also presented graphically in table.1.

Table 1: Entropy and PSNR values

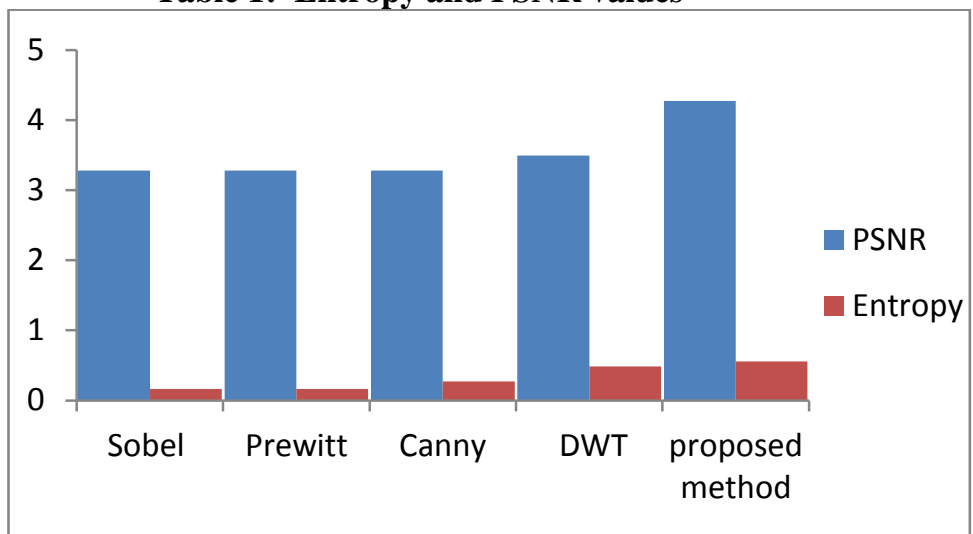

Table 2: Comparison of Entropy and PSNR values

\begin{tabular}{|c|l|l|l|}
\hline No. & Algorithm & Entropy & PSNR \\
\hline $\mathbf{1 .}$ & Edge detection using Sobel & 0.16491 & 3.27801 \\
\hline $\mathbf{2 .}$ & Edge detection using Prewitt & 0.16463 & 3.27803 \\
\hline $\mathbf{3 .}$ & Edge detection using Canny & 0.27261 & 3.27811 \\
\hline $\mathbf{4 .}$ & Edge detection using DWT & 0.48741 & 3.4934 \\
\hline
\end{tabular}




\begin{tabular}{|l|l|l|l|}
\hline 5. & $\begin{array}{l}\text { Edge detection using Proposed } \\
\text { Method }\end{array}$ & 0.55344 & 4.5759 \\
\hline
\end{tabular}

\section{CONCLUSION}

It is shown that the proposed technique when implemented gives better result than the comparative method. Proposed method compared with the Prewiit, Canny, Sobel as well as DWT. Experimental result shows that the proposed algorithm works more efficiently. It has been observed that discrete wavelet transform in two dimensions have limited ability in capturing dimensional information. Proposed work also removes the problem of not getting continuous edges is being solve. In future comparison can be done by taking more parameters. Fuzzy logic type -2 can be used as an extension to the project

\section{ACKNOWLEDGMENTS}

This paper is part of the project implementation. Authors thank to Dr. M. V. Deshpande, Associate Dean, Mukesh Patel School of Technology Management \& Engineering, NMIMS, Shirpur, for his valuable guidance.

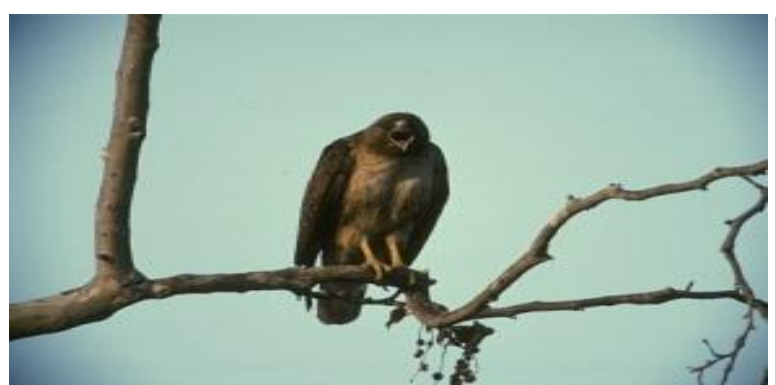

(a)

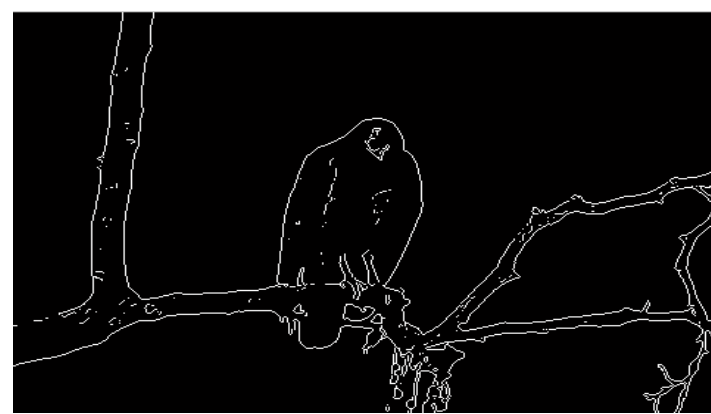

(c)

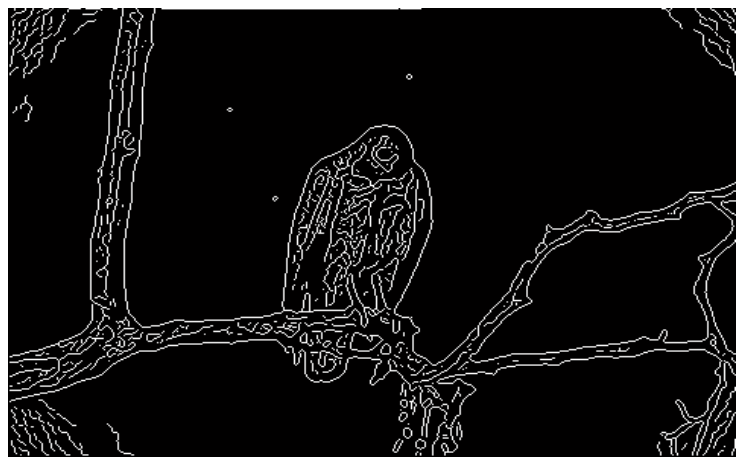

(e)

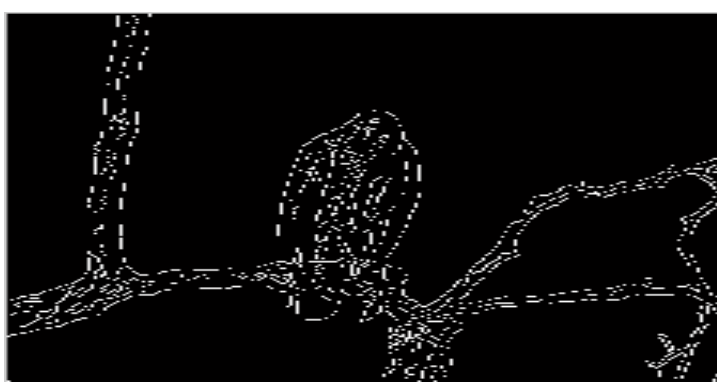

(b)

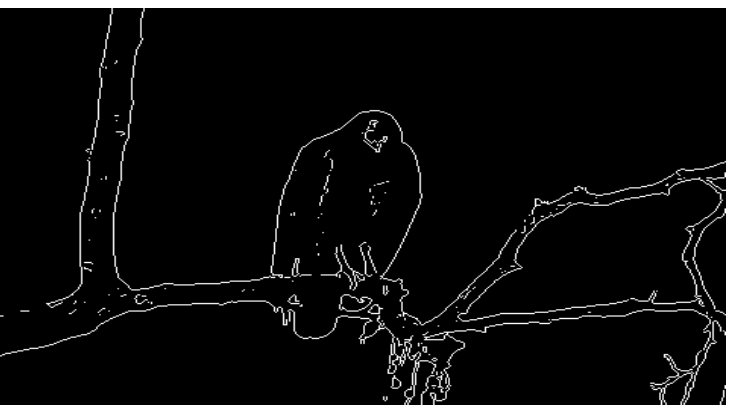

(d)

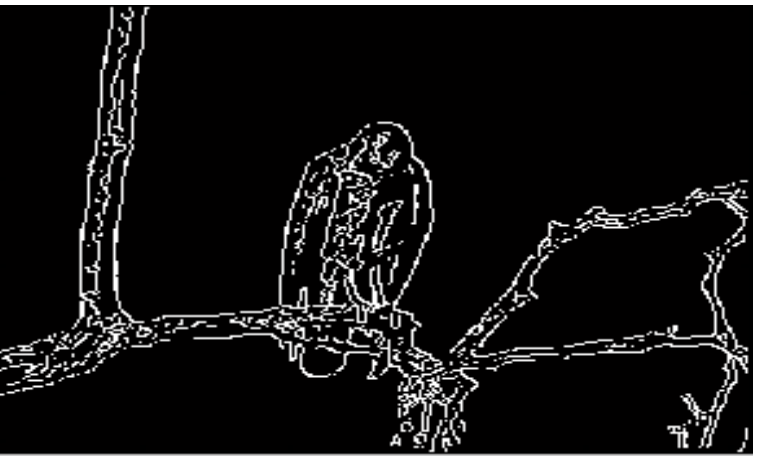

(f) 


\section{REFERENCES}

[1] Rafael C. Gonzalez, Richard E. Woodes, and Steven L. Eddins, DIGITAL IMAGE PROCESSING, published by Pearson Education (Singapore) Pvt. Ltd

[2] Dr. H. B. Kekre and Ms. Saylee M. Gharge, "Image Segmentation using Extended Edge Operator for Mammography Images", International Journal on Computer Science and Engineering, Vol.02, No. 04, 2010, 1086-1091.

[3] Y. Becerikli and H. EnginDemiray, Alternative Neural Network Based Edge Detection, Neural Information Processing, Vol. 10, Nos.8-9, Sept. 2006.

[4] M. Egmont-Petersen, D. de Ridder, H. Handels, Image processing with neural networks - a review, Vol. 35, No. 10, pp. 2279-2301,2002.

[5] J. Canny, "A Computational Approach to Edge Detection", IEEE Transactions on Pattern Analysis and Machine Intelligence. 8 (6), pp- 679-687, 1986.

[6] M. Zhao, A. M .N .Fu, H. Yan, "a technique of three Level Thresholding Based on Probablity Partition an Fuzzy 3-Partition", IEEE Trans on Fuzzy System,vol.9, no.3, pp.469-479, June 2001

[7] S. Wang, F. Ge, T. Liu, "Evaluating Edge Detection through Boundary Detection", Department of Computer Science and Engineering, Univercity of south Carolina,Columbia, Usa, 2006.

[8] W. zhang, J, Kang, "Edge detection based on fusion of wavelet transform and mathematical morphology", IEEE, 2009.

[9] Serra, J.: 'Image analysis and mathematical morphology' (Academic, New York, 1982)

[10] Samet, H., and Webber, R.E.: 'Hierarchical data structures and algorithms for computer graphics.I. Fundamentals', IEEE Trans. Comput. Graph. Appl., 1988, 8, (3), pp. 48-68)

[11] Yang Yong, Huang Shuying, Modified Pal and King algorithm for fuzzy edge detection, Chinese Journal of Scientific Instrument, Vol. 29, No.9, 2008, pp. 19181922

[12] Zhang Jinping, Lian Yongxiang, Dong Linfu, A new method of fuzzy edge detection based on Gauss function, International Conference on Computer and Automation Engineering (ICC AE), Vol.4, 2010, pp. 559-562.

[13] Fabrizio Russo "Edge Detection in Noisy ImagesUsing Fuzzy Reasoning" IEEE Instrumentation and Measurement Technology Conference St.Paul, Minnesota, USA, May 18-21, 1998 Edge Detection in Noisy ImagesUsing Fuzzy Reasoning.

[14] Yan Ha "Method of edge detection based on Non-linear cellular Automata" 978-1-4244-2114-5/08/\$25.00 (C) 2008 IEEE

[15] Gao Qinqing ,Chen Dexi ,ZengGuangping and He Ketai* "Image Enhancement Technique Based On Improved PSO Algorithm" 978-1-4244-8756-1/11/\$26.00_c 2011 IEEE

[16] Mrs.Abhradita Deepak Borkar and Mr.MithileshAtulkar "Detection of Edges Using Fuzzy Inference System" International Journal of Innovative Research in
Computer and Communication Engineering Vol. 1, Issue 1, March 2013

[17] S. Mallat, W. L. Hwang, "Singularity detection and processing with wavelet," IEEE Tran. Inform.Theory, Vol. 38, No. 2, March, 1992.

[18] S. Wenchang, S. Jianshe, Z. Lin, "Wavelet Multi-scale Edge Detection Using Adaptive threshold," IEEE, 2009.

[19] L. Zhang, P. Bao, "Edge detection by scale multiplication in wavelet domain," Elsevier Science B.V. Pattern Recognition letters 23, pp. 1771-1784, 2002.

[20] C. Q. Zhan, L. J. Karam, "Wavelet-based adaptive image denoising with edge preservation," IEEE, 2003.

[21] E. Brannock, M. Weeks, "Edge detection using wavelets," ACM SE, March, 2006.

[22] Masrour Dowlatabadi and Jalil Shirazi," Improvements in Edge Detection Based on Mathematical Morphology and Wavelet Transform using Fuzzy Rules" World Academy of Science, Engineering and Technology Vol:5 2011-10-22

[23] ArpitSinghal, Mandeep Singh "Speckle Noise Removal and Edge Detection Using Mathematical Morphology" International Journal of Soft Computing and Engineering (IJSCE) ISSN: 2231-2307, Volume-1, Issue- 5, November 2011

[24] Mehul Thakkar and Prof. Hitesh Shah "Edge Detection Techniques Using Fuzzy Thresholding" 978-1-46730126-8/11/\$26.00c 2011 IEEE

[25] Zhao Yuqian, Gui Wei-hua, Chen Zhencheng, Tang Jingtian, and Li Ling-yun., "Medical Images Edge Detection Based on Mathematical Morphology", ", Proceedings of the IEEE Engineering in Medicine and Biology 27th Annual International Conference Shanghai, China, PP: 6492-6495, 2005.

[26] J Patel and et al., "Fuzzy Inference based Edge Detection System using Sobel and Laplacian of Gaussian Operators" ICWET'11, ACM 978-1-4503-0449-8, pp 694-697, February 25-26, 2011

[27] Abdallah A. Alshennawy, and Ayman A. Aly, "Edge Detection in Digital Images Using Fuzzy Logic Technique", World Academy of Science, Engineering and Technology, 2009, pp-178-186.

[28] Pushpajit A. Khaire and Nileshsingh V. Thakur. "Image Edge Detection based on Soft Computing Approach" International Journal of Computer Applications (0975 8887) Volume 51- No.8, August 2012

[29] Pushpajit A. Khaire and Nileshsingh V. Thakur, "A Fuzzy Set Approach for Edge Detection" International Journal of Image Processing (IJIP), Volume (6): Issue (6), 2012

[30] L.R. Liang, C.G. Looney," Competitive fuzzy edge detection", Applied Soft Computing, Vol.3, No.2, 2003, pp. 123-137.

[31] RajasekaranS and G A VijayalakshmiPai, Neural Networks, Fuzzy Logic and Genetic AlgorithmsSynthesis and Applications, Prentice-Hall of India, 2003

[32] SamanSinaie, AfshinGhanizadeh, and SitiMariyam Shamsuddin and EmadaldinMozafariMajd "A Hybrid Edge Detection Method Based on Fuzzy Set Theory and 
CellularLearning Automata" 2009 International Conference on Computational Science and Its Applications 978-0-7695-3701-6/09 \$25.00 (c) 2009 IEEE DOI 10.1109/ICCSA.2009.19

[33] Dhirajkumar Patel and S A More "Edge Detection Technique by Fuzzy Logic and Cellular Learning Automata using Fuzzy Image Processing" 978-1-46732907-1/13/\$31.00 @2013 IEEE

[34] Dhirajkumar Patel and S A More "An enhanced approach for EDGE image enhancement using fuzzy set theory and cellular learning automata (CLA)" World Journal of Science and Technology 2012, 2(4):158-162

[35] Hamid R. Ezhyosh, "Fast Fuzzy Edge Detection" 07803-7461\%/02 pp.239-242 IEEE 2002
[36] Raman Maini and HimanshuAggarwal, "Study and Comparison of Various Image Edge Detection Techniques", International Journal of Image Processing (IJIP), Volume (3), 2010, pp-1-12.

[37] Li Fang, Weiren Shi, Shuhan Chen," Fuzzy reasoningbased edge detection method using multiple features" WSEAS TRANSACTIONS on COMPUTERS E-ISSN: 2224-2872 Issue 11, Volume 11, November 2012

[38] Y. Xu et al, “ Wavelet transform domain filter: a spatially selective noise filtration technique", IEEE Trans on image processing, vol..3, pp.747- 758, 1994.

[39] Chaganti, Venkata Ravikiran, "Edge Detection of Noisy Images using 2-d Discrete Wavelet Transform" (2005) Electronic Theses, Treatises and Dissertations. Paper 3948. 